International Journal of BioAnalytical Methods \& BioEquivalence Studies (JJBMBS)

ISSN 2470-4490

\title{
Sterilization Mechanism of Nitrogen Gas Plasma Exposure
}

\section{Editorial}

Hideharu Shintani

Faculty of Science and Engineering, Chuo University, 1-13-27, Kasuga, Bunkyo, 112-8551, Tokyo, Japan.

\section{*Corresponding Author:}

Hideharu Shintani,

Faculty of Science and Engineering, Chuo University, 1-13-27, Kasuga, Bunkyo, 112-8551, Tokyo, Japan.

E-mail: shintani@mail.hinocatv.ne.jp

Received: March 07, 2015

Published: March 09, 2015

Citation: Hideharu Shintani (2015) Sterilization Mechanism of Nitrogen Gas Plasma Exposure. Int J Bioanal Methods Bioequival Stud, 2(1e).1-2. doi: http://dx.doi.org/10.19070/2470-4490-150001e

Copyright: Hideharu Shintani ${ }^{\circ}$ 2015. This is an open-access article distributed under the terms of the Creative Commons Attribution License, which permits unrestricted use, distribution and reproduction in any medium, provided the original author and source are credited.

As already published by Shintani et al, gas plasma sterilization is the alternative sterilization procedures to the existing sterilization procedures due to applicable to the several sensitive healthcare products and attainable both sterility assurance level (SAL) of $10^{-6}$ and material/functional compatibility, which authorities and GMP (good manufacturing practice) are required. In addition there was progress in the field of plasma-based sterilization, there are still some questions. The aim of this editorial is to mention briefly some of un-resolved sterilization mechanisms as well as to draw further perspectives of sterilization technique.
The atmospheric- and low-pressure gas plasma can be used to sterilize bioburden in the surfaces. Several mechanisms have been clearly identified, namely reaction with reactive species as shown in Figures 1-2 were mostly the major contributors. The speculated major contributor was peroxynitrite anion. Nitric monooxide radical detected by us [3] combines with superoxide anion and produce peroxynitrite anion on the biological indicator spores or bioburden to sterilize them. Life period of the peroxynitrite anion, nitric monoxide radical and superoxide anion was a few s, 3-6 $\mathrm{s}$ and $5 \mathrm{~s}$, respectively, which are relatively long period compared with most of radicals with $\mu$ s life period such as $\mathrm{OH}$ radical. As the outer layers of gram-negative and gram-positive bacteria have been charged (Figure 3), therefore charged contributors cannot penetrate into the interior of the bacteria to attack interior DNA or RNA. In that sense, nitric monoxide radical can be understood due to neutral, but superoxide anion has a problem to understand due to minas charge. As a whole speculation of peroxynitrite anion was problematic to define and required to consider another reasonable factor to consider without problems. As being indicated charged factors can be neglected as contributors. This indicates that metastables of $\mathrm{N}$ or $\mathrm{O}$ may be the major contributors due to a few s of life period, neutral and abundant energy produced from the excited state to the ground state emitted energy to destroy bacteria. Radicals may be candidate as they are not charged, but their life period is too short such as a few $\mu \mathrm{s}$, so radicals with long life period may be the candidates. In that means $\mathrm{NO}$ radical with 3-6 s of life period may also be candidate, but unfortunately NO

Figure 1. Model case of gas plasma exposure.

Cited from http://www.astp.com/plasma-equipment/applications

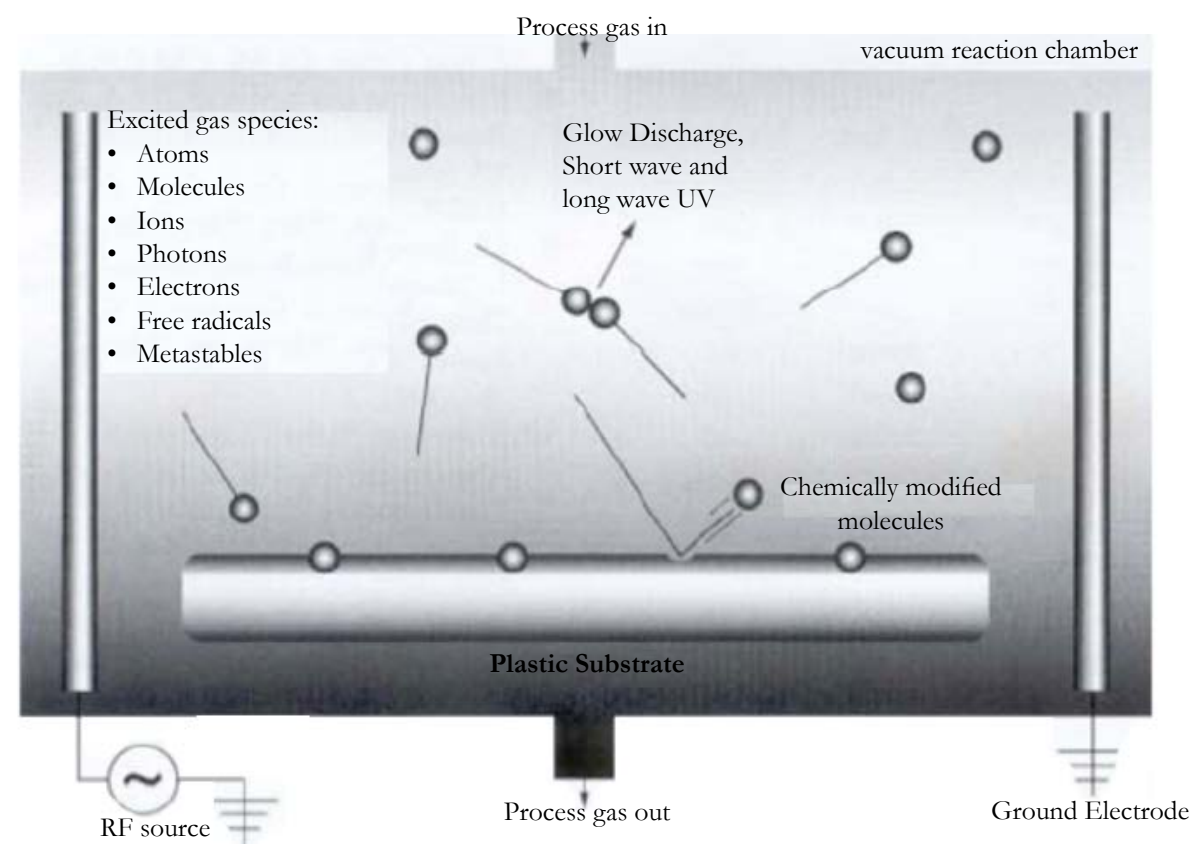


Figure 2. Example of oxygen gas plasma exposure.

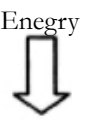

Gas $\left(0_{2}\right)$

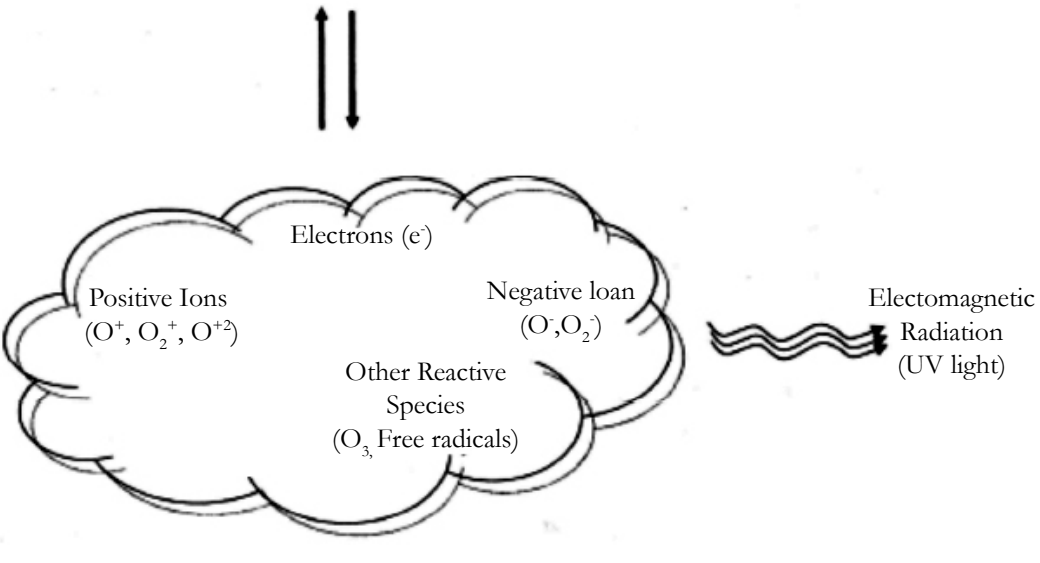

Plasma

Figure 3. Outer layer of gram negative and gram positive bacteria.

Gram positive

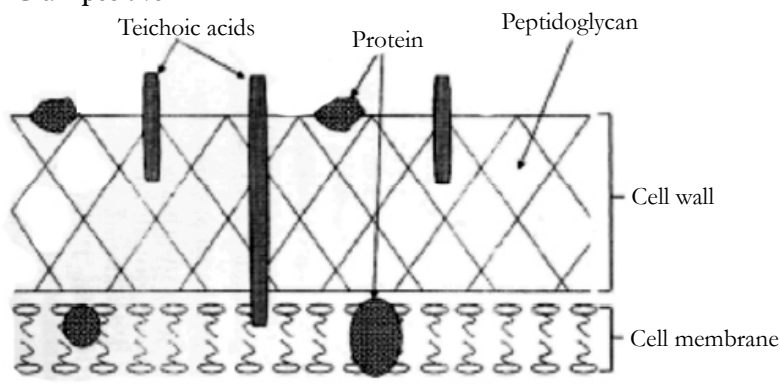

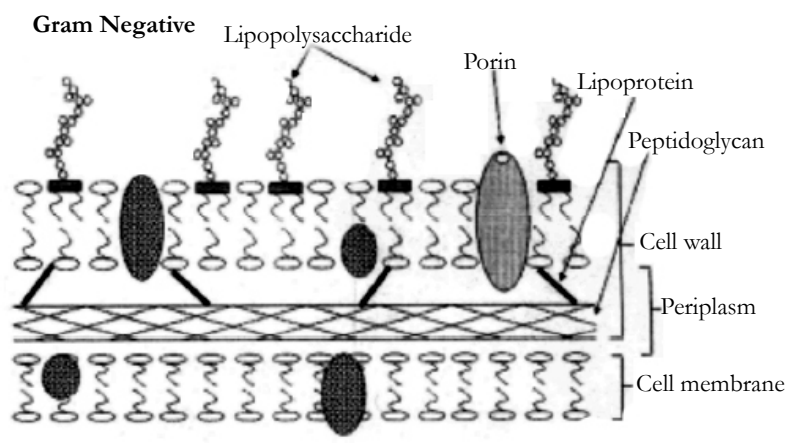

radical has no sterilization or disinfection function.

UV leads to very effective sterilization when spores are not clumped on the materials. However, UV or VUV alone is not efficient for inactivating bacterial spores, endotoxin or prion proteins, indicating gas plasma produce UV or VUV (Figures 1 and 2), but they are not completely major contributors.

Apart from the problems related to the matrix effect, another practical difficulty in the use of plasma discharges is related to their directionality and their limited penetration at $10-20 \mathrm{~nm}$ in high aspect ratio holes and trenches (1ow-pressure plasma discharges, [1, 2]). The former requires special handling of the equipment during treatment, which complicates the operation. The latter requires the procedure to be carried out at intermediate pressures: this limits the efficiency of the chemical sputtering effect, which decreases the sterilization effects.
Finally, plasma treatments are not very compatible with pre-packaged instruments, as the packaging may block the reactive species created by the plasma discharge. This is particularly crucial for atmospheric plasma treatment, since at low pressure, the plasma discharge could in principle be created inside the package, which means gas plasma sterilization is not carried out like $\gamma$-ray or ebeam irradiation sterilization. This is seriously obstacle to apply the gas plasma sterilization for the practical use in future.

\section{References}

[1]. Shintani H, Shimizu N, Imanishi Y, Sekiya T, Tamazawa K, et al.(2007) Inactivation of microorganisms and endotoxins by low temperature nitrogen gas plasma exposure. Biocontrol Science 12(4): 131-143.

[2]. Shintani H (2012) Inactivation of prion and endotoxins by nitrogen gas plasma exposure. Pharmaceutica Analytica Acta 3 (8): 177. http://dx.doi. org/10.4172/2153-2435.1000177

[3]. Shintani H, Saito T, Nakamur K (2014) Chemical indicator and ink constitute to detect several radicals, metastables and ions produced by gas plasma sterilization. Japan Patent 233387. 